\title{
Study of Survivin: A Biomarker in Carcinoma Colon - An Original Study
}

\author{
Pratibha Chandra and Prateek Kinra* \\ Department of Pathology, AFMC, Pune
}

\begin{abstract}
Background: Survivin, an anti-apoptotic protein has been of importance in colon carcinoma patient. Here we studied the prevalence of Survivin biomarker among cases of colon carcinoma and its relationship with other prognostic indicators.

Design: Tissue samples from 102 patients with colon carcinoma were studied. H\&E stain and immunohistochemistry were performed and evaluated. Immuno-histochemistry expression was sought according to IRS Scoring System (Immunoreactivity Scoring) for stain intensity and percentage positive cells. Survivin expression was also correlated with age at diagnosis, gender, grade, nodal status, lympho-vascular and perineural invasion, resection margins, Tumor stage (TNM) and Group Stage.

Result and Discussion: Survivin was expressed in high grade and stage tumors and showed significant association ( $p$ value $=0.002)$. Survivin expression was positive in lymph node positive tumors and showed significant association ( $\mathrm{p}$ value $=0.002$ ). Survivin was expressed in tumors with lympho-vascular and perineural invasion and showed significant association ( $\mathrm{p}$ value $=0.02$ and 0.001 ) respectively. Age at diagnosis showed significance of $\mathrm{p}=0.03$ whereas Gender (Male/Female) and resection margins did not show any association with Survivin expression.

Conclusion: Survivin expression increases as the tumor grade worsens in colon cancer and is maintained in approximately all high group staged tumors (TNM Staging) along with tumors showing lymphovascular and perineural invasion. Gender does not have any association.
\end{abstract}

Keywords: Survivin, Carcinoma Colon, Antiapoptotic Protein, Immunoreactivity Scoring System.

\section{Introduction}

Colorectal cancer is a daunting health problem worldwide. It is the third most common cancer in men and the second most common in women. ${ }^{[1]}$ In present clinical practice, colon cancer has been prognosticated using following variables: (i) stage and grade of tumor, (ii) lymph and bloodvessel invasion, (iii) perineural invasion(PNI), (iv)Tumor budding, (v)Tumor necrosis, (vi) Inflammatory response. ${ }^{[2]}$ Tumor staging according to the American Joint Committee on Cancer/Union for International Cancer Control tumor, node, metastasis (TNM) system is currently regarded as the standard for staging of patients with colorectal cancer. This system provides the strongest prognostic information for patients with early stage disease and those with advanced disease. For patients with intermediate levels of disease, it is less able to predict disease outcome. Therefore, additional prognostic markers are needed to improve the management of affected patients..$^{[3]}$

Survivin, also called baculoviral inhibitor of apoptosis repeat-containing 5 or $\mathrm{BIRC} 5$, is a protein that, in humans, is encoded by the BIRC5 gene and is a member of the inhibitor of apoptosis family (IAPs). The survivin protein functions to inhibit caspase activation, thereby leading to negative regulation of apoptosis or programmed cell death. This has been shown by disruption of Survivin induction pathways leading to increase in apoptosis and decrease in tumour growth. The survivin protein is expressed highly in most human tumours and fetal tissue, but is completely absent in terminally differentiated cells. It might provide a new target for cancer therapy that would discriminate between transformed and normal cells. Survivin expression is also highly regulated by the cell cycle and is only expressed in the G2-M phase. It is known that survivin localizes to the mitotic spindle by interaction with tubulin during mitosis and may play a contributing role in regulating mitosis. ${ }^{[4]}$ Survivin is one of the top five tumor markers, exclusively overexpressed in most cancers, making it an ideal target for cancer therapeutics. High level of survivin help in the promotion of cancer development through contributing via a wide range of cellular mechanisms including growth and apoptotic pathways. In cancer patients, an elevated level of survivin is often associated with poor prognosis and therapy resistance, and it also promotes metastasis in cancer cells. Several gene silencing studies have clearly demonstrated a crucial role of survivin in cancer development. ${ }^{[4]}$

Currently, most of the cancer treatment protocols are an involved combination of surgery, chemotherapy and radiotherapy, but even after substantial growth in this direction, patient survival rates have not changed much. 
Therefore, lots of studies are being conducted to explore the possibility of using molecular targeting therapies along with conventional therapies to tackle the menace of cancer. An increasing number of clinical trials are taking place with the selected patients based on validated biomarkerenrichment. Survivin is considered an excellent molecular target for cancer treatment, and several therapeutic strategies, such as gene silencing, immunotherapy, and small molecule inhibition have been designed and tested in different pre-clinical and clinical studies. In the future, administration of survivin-targeted agents alone or in combination with conventional therapies may generate a novel therapeutic strategy against cancer. ${ }^{[5]}$

\section{Material and Methods}

The study was carried out in the tertiary care centre. Study population included all the diagnosed cases of Colon Cancer who had undergone definitive surgery in last five years. Only colectomy specimen included which enabled us to correlate with all known prognostic variables (Inclusion criteria). All endoscopic biopsy, colonic stromal tumors, neuroendocrine tumors and lymphoma are excluded (Exclusion criteria). The institutional ethical committee clearance for the present study was obtained in the month of November 2017 at the time of submission of synopsis to the Maharashtra University of Health Sciences (MUHS), Nashik. Informed consent was taken from all the patients whose specimens were received in our department post undertaking of the study. However, the consent could not be taken in the cases taken retrospectively. The formalin fixed paraffin embedded (FFPE) tissue blocks were retrieved from the departmental archives for the retrospective cases. The clinical data was recorded from the database of the department and wherever required. For prospective tumors, the post-operative resection specimens were collected in formalin containing labelled containers. College of American Pathologists (CAP) protocol was followed for grossing the specimens. Representative tumor sections were taken and submitted for histopathological examination. We assessed the cases for various variables like age at diagnosis, gender, differentiation, nodal status, lymphovascular invasion (LVI), perineural invasion (PNI), stage and resection margin status along with H\&E stained sections and immunohistochemistry expression profiles of patients with colonic adenocarcinoma for the expression of Survivin. Immunohistochemistry (IHC) expression was sought according to IRS Scoring System (Immunoreactivity Scoring) for stain intensity and percentage positive cells. Tumors were staged according to the AJCC criteria, 8th edition. ${ }^{[7]} 102$ controls were selected from unaffected portion of the resected specimens of conditions like ischemic colitis, colonic perforation.
Immunoreactive scoring system (IRS): Survivin expression in the cytoplasm and in the nucleus is grouped in to four groups: Group 1: negative (IRS 0 to 2); Group 2: weak (IRS 3 to 5); Group 3: moderate (IRS 6 to 8); Group 4: strong (IRS 9 to 12). Semi-quantitative assessment of Survivin expression is based on the mean percentage of positive tumour cells in at least five high-power fields $(\times 400)$ assigned to one of five categories: (a) $0=<5 \%$; (b) $1=5-25 \%$; (c) $2=25-50 \%$; (d) $3=50-75 \%$; (e) $4=>75 \%$. The intensity of Survivin immunostaining is scored as (a) weak $=1+$; (b) moderate $=2+$; (c) intense $=3+$. The percentage of positive tumour cells and staining intensity are multiplied to produce a weighted score for each case. In tumours displaying heterogeneous staining, the predominant pattern is considered for scoring. Tumours with a weighted score $=0$ are designated as negative; all others are considered positive. ${ }^{[6]}$

Statistical Analysis: Student $t$ test was used to assess the difference in Survivin IHC expression in cancerous tissue for age at diagnosis. Mean values with standard errors were used to visualize different categories. Chi square test was used to test whether categorical parameters (e.g. grade, gender, stage, LVI, PNI, Nodal status) were associated with Survivin status.

\section{Results}

We studied 102 consecutive resection specimens of colorectal carcinomas from January 2014 to July 2019. We had 102 cases and 102 healthy controls. Out of 102 cases, as per immunohistochemical expression of Survivin marker, we divided the specimens as Survivin positive or negative. 26 cases were Survivin positive cases and 76 were Survivin Negative cases. 102 healthy controls were studied by doing H\&E stain followed by immunohistochemistry for Survivin. Out of all controls, none of the case showed positivity for Survivin in nucleus or cytoplasm.

The age of the patients ranged from 21 years to 84 years with a mean age of 60.62 years. The maximum number of patients were in the age group of 61-70 years which accounted for 32 cases $(31.37 \%)$, closely followed by 23 $(22.54 \%)$ patients in age group of $71-80$ years. This study comprised of a total of 74 males and 28 females. This is in accordance with the general trend across the globe of colorectal carcinomas being more predominant in the male gender.

Age at diagnosis: Of all the 102 colorectal carcinomas, 27 patients $(26.47 \%)$ were less than or equal to 50 years old at the time of diagnosis and the rest $(73.52 \%)$ were 75 above the 50 years bracket. This association was statistically significant with a $p$ value of 0.014 and a $t$-value of -2.49 . 
Out of 26 Survivin positive cases, $57.69 \%$ cases were more than 50 yrs age whereas $42.3 \%$ cases belonged to less than 50yrs. (Fig No. $1 \&$ Table No.1)

Gender: Out of 26 Survivin positive cases, $69 \%$ were male and $30.7 \%$ were female. However, this correlation was not statistically significant with the $p$ value for this test of association being 0.66.(Fig No. $2 \&$ Table No.1)

Grade of tumor/differentiation: The tumor was graded as well, moderately and poorly differentiated based on the glandular differentiation. Out of Survivin positive tumors $(\mathrm{n}=26), \quad 34.61 \%$ cases belonged to poorly differentiated grade whereas $15.38 \%$ to well differentiated and $53.84 \%$ to moderately differentiated grade.(Fig No. 3, Table No. 1) However, association of this variable was statistically significant to the occurrence of Survivin Positivity ( $p$ value $=0.0009$ ). Inference of this value is that as the grade increases, positivity for Survivin increases.

Nodal status: Out of 26 Survivin positive cases, $65.38 \%$ cases had lymph node spread whereas $34.61 \%$ cases did not have nodal spread. The statistics showed statistically significant $\mathrm{p}$ value $(p$ value $=0.002)$. This value infers that the tumors with higher lymph node spread showed more Survivin positivity.

Lymphovascular invasion (LVI): Out of 76 Survivin negative cases, $82.89 \%$ cases did not have lymphovascular invasion whereas only $17.10 \%$ cases had lymphovascular invasion. Association of this variable was statistically significant to the occurrence of Survivin Positivity $(P$ value $=0.0089$ ). This value infers that the tumors with lymphovascular invasion have higher chance of showing Survivin positivity.

Perineural invasion (PNI): Of the 102 patients, 11 patients $(10.78 \%)$ had PNI out of which 10 were positive for Survivin. While 91 patients $(89.21 \%)$ didn't have any PNI. Association of this variable was highly statistically significant to the occurrence of Survivin Positivity ( $P$ value $=0.002$ ). We can construe that the tumors with no perineural invasion have less chance of showing Survivin positivity.

Group Staging: The Group Staging was categorized as I and II in one group (low stage) whereas III and IV in other group (high stage). Of the 102 patients, 61 patients (59.8\%) were in low stage group out of which 9 were positive for Survivin. While 41 patients $(40.19 \%)$ are grouped under high stage group out of which 17 were survivin positive. Association of this variable was statistically significant to the occurrence of Survivin positivity $(\mathrm{P}$ value $=0.002$ ) This analysis confers that the tumors with high stage showed more Survivin positivity.
Resection margin: Of the 102 patients, only 01 patients $(0.98 \%)$ had positive margin and was positive for Survivin. While 101 patients $(99.01 \%)$ were margin negative. Of the 101 patients, 25 cases were Survivin Positive. Since there was only a single case, the odds and $p$ value could not be calculated in this case.

A univariate analysis of all the clinico-pathologic variables in tumors with and without Survivin positivity was done (Table 1). The univariate analysis in our index study showed the following variables to be associated with the occurrence of Survivin Positive tumors with a $p$ value of $<0.05$ : Age at diagnosis, Grade of tumor, Lymph node spread, Lymphovascular - invasion, Perineural invasion, Group Stage.

Multivariate analysis was done on the significant parameters using binary logistic regression and was not significant (Table 2). The results were compared with other studies (Table 3 ) conducted in the past. ${ }^{[8,9]}$ Sarela et $a^{\left[{ }^{[8]}\right.}$ concluded that the lymph node spread (Table 4) was significantly associated with the occurrence of Survivin positivity whereas there was no significance with respect to age (Table 5), gender (Table 6) and group stage ( Table 7). Grade of the tumor, LVI and PNI were not studied. Kawasaki et $\mathrm{a}^{\left[{ }^{[9]}\right.}$ concluded that the following parameters were significantly associated with the occurrence of Survivin positivity: (a) Tumor depth, (b) Peritoneal metastases, (c) Hepatic metastases whereas there was no significance in respect of age, gender, grade, LVI and group stage. Perineural invasion was not studied. Resection margin was not studied in both the studies.

\section{Discussion}

Colorectal adenocarcinomas are amongst the common malignancies of the gastrointestinal tract causing tremendous amount of morbidity and mortality among the sufferers. With the advancement in the understanding of the pathology and evolution of the disease, more and more targeted approach has come in light with regards to the management of the disease. TNM system is currently regarded as the standard for staging of patients with colorectal cancer. This system provides the strongest prognostic information for patients with early stage disease and those with advanced disease. For patients with intermediate levels of disease, it is less able to predict disease outcome. Therefore, additional prognostic markers are needed to improve the management of affected patients. ${ }^{[3]}$

A number of independent prognostic factors are currently being evaluated in nomograms that also include TNM stage of disease. In the future, the intratumoral expression of specific molecules, e.g., Deleted in Colorectal Cancer (DCC) or 18q loss of heterozygosity (LOH), p27 Kip1, 
Table 1: Univariate analysis of all the variables in Survivin Positive and Survivin Negative colorectal carcinomas.

\begin{tabular}{|c|c|c|c|c|c|}
\hline Features & $\begin{array}{l}\text { Survivin(+) } \\
(\mathrm{n}=26)\end{array}$ & $\begin{array}{l}\text { Survivin (-) } \\
(n=76)\end{array}$ & Total & Odds Ratio & $p$ Value \\
\hline \multicolumn{5}{|l|}{ Age at diagnosis } & \multirow[t]{5}{*}{0.0014} \\
\hline$<50 \mathrm{yrs}$ & 11 & 16 & 27 & \multirow[t]{2}{*}{2.75} & \\
\hline$>50 \mathrm{yrs}$ & 15 & 60 & 75 & & \\
\hline Mean & 54.76 years & 57.57years & - & - & \\
\hline SD & 8.53 & 8.19 & - & - & \\
\hline \multicolumn{5}{|l|}{ Gender } & \multirow[t]{3}{*}{$0.66^{*}$} \\
\hline Male & 18 & 56 & 74 & \multirow[t]{2}{*}{0.80} & \\
\hline Female & 8 & 20 & 28 & & \\
\hline \multicolumn{5}{|l|}{ Grade(Differentiation) } & \multirow[t]{3}{*}{0.0009} \\
\hline Poorly & 9 & 6 & 15 & \multirow[t]{2}{*}{6.18} & \\
\hline Well \& Moderately & 17 & 70 & 87 & & \\
\hline \multicolumn{5}{|l|}{ Lymph node status } & \multirow[t]{3}{*}{0.002} \\
\hline Positive & 17 & 24 & 41 & \multirow[t]{2}{*}{4.09} & \\
\hline Negative & 61 & 9 & 52 & & \\
\hline \multicolumn{5}{|l|}{ LVI } & \multirow[t]{3}{*}{0.0089} \\
\hline Present & 11 & 13 & 24 & \multirow[t]{2}{*}{3.55} & \\
\hline Absent & 15 & 63 & 78 & & \\
\hline \multicolumn{5}{|l|}{ PNI } & \multirow[t]{3}{*}{0.001} \\
\hline Present & 10 & 1 & 11 & \multirow[t]{2}{*}{46.8} & \\
\hline Absent & 16 & 75 & 91 & & \\
\hline \multicolumn{5}{|l|}{ Group Stage } & \multirow[t]{3}{*}{0.002} \\
\hline Low Stage (I \& II) & 9 & 52 & 61 & \multirow[t]{2}{*}{0.244} & \\
\hline Hight Stage (III \& IV) & 17 & 24 & 41 & & \\
\hline \multicolumn{5}{|l|}{ Resection Margin } & \multirow[t]{3}{*}{ NA } \\
\hline Positive & 01 & - & 01 & \multirow[t]{2}{*}{ NA } & \\
\hline Negative & 25 & 76 & 101 & & \\
\hline
\end{tabular}

Table 2: Multivariate analysis by using Binary Logistic regression with outcome variable Survivin +/- with various predictors (B - coefficient; S.E - Standard error; d.f. - degree of freedom) (* - Not statistically significant).

\begin{tabular}{|l|l|l|l|l|l|l|l|}
\hline Predictors of Survivin & B & SE & d.f. & P value & Odds ratio & 95\% Cl & Upper \\
\cline { 5 - 9 } & & & & & & Lower & ( \\
Age at diagnosis & -0.039 & 0.019 & 1 & 0.041 & 0.962 & 0.927 & 0.998 \\
\hline Gender & -0.039 & 0.581 & 1 & 0.947 & 0.962 & 0.308 & 3.005 \\
\hline Grade & 0.332 & 0.520 & 1 & 0.524 & 1.393 & 0.503 & 3.860 \\
\hline Lymph Node & - & - & 1 & - & - & - & - \\
\hline LVI & -0.881 & 0.716 & 1 & 0.219 & 0.415 & 0.102 & 1.686 \\
\hline PNI & -0.892 & 0.907 & 1 & 0.326 & 0.410 & 0.069 & 2.427 \\
\hline Group Stage & 0.896 & 0.537 & 1 & 0.095 & 2.450 & 0.855 & 7.017 \\
\hline Resection margin & - & - & - & NA & - & - & - \\
\hline
\end{tabular}


Table 3: Statistical association of variables associated with occurrence of Survivin Positivity (univariate analysis).

\begin{tabular}{|l|l|l|l|}
\hline S.No. & Index Study & Sarela et al ${ }^{\left[{ }^{[]}\right.}$ & Kawasaki et al ${ }^{[9]}$ \\
\hline 1.Age at diagnosis & Significant & Not significant & Not significant \\
\hline 2.Gender & Not significant & Not significant & Not significant \\
\hline 3.Grade & Significant & - & Not significant \\
\hline 4.Lymphnode spread & Significant & Significant & Not significant \\
\hline 5.Lymphovascular invasion & Significant & - & Not significant \\
\hline 6.Perineural invasion & Significant & - & - \\
\hline 7.Group Stage & Significant & Not Significant & Not significant \\
\hline Resection margin & NA & - & - \\
\hline
\end{tabular}

Table 4: Comparing the variable "Lymph node status" with other studies.

\begin{tabular}{|l|l|l|l|}
\hline Grade of tumor & Index Study & Sarela et Al[8] & Kawasaki et al[9] \\
\hline Odds ratio & 4.09 & - & $0.93-2.45$ \\
\hline $95 \% \mathrm{Cl}$ & $3.15-5.03$ & $1.33-5.11$ & 0.0833 \\
\hline$p$ value & 0.002 & 0.49 & \\
\hline
\end{tabular}

Table 5: Comparing the variable "Age at diagnosis" with other studies.

\begin{tabular}{|l|l|l|l|}
\hline Age at diagnosis & Index study & Sarela et al[ ${ }^{[]}$ & Kawasaki et al[9] \\
\hline Odds ratio & 2.75 & - & - \\
\hline $95 \% \mathrm{Cl}$ & $1.88-3.37$ & $0.57-2.92$ & - \\
\hline Mean age of occurrence & 57.57 years & - & 59.7 \\
\hline SD & 14.19 & - & 12 \\
\hline p Value & 0.014 & 0.49 & 0.82 \\
\hline
\end{tabular}

Table 6: Comparing the variable "Female sex" with other studies.

\begin{tabular}{|l|l|l|l|}
\hline Female Sex & Index Study & Sarela et al ${ }^{[8]}$ & Kawasaki et al $^{[9]}$ \\
\hline Odds ratio & 0.8 & - & - \\
\hline $95 \% \mathrm{Cl}$ & $0.17-1.78$ & $0.32-1.41$ & - \\
\hline $\mathrm{P}$ value & 0.66 & 0.49 & 0.23 \\
\hline
\end{tabular}

Table 7: Comparing the variable "Group Stage" with other studies.

\begin{tabular}{|l|l|l|l|}
\hline Grade of tumor & Index study & Sarela et al ${ }^{[8]}$ & Kawasaki et al $^{[9]}$ \\
\hline Odds ratio & 0.244 & - & - \\
\hline $95 \% \mathrm{Cl}$ & $0.69-1.18$ & - & - \\
\hline$p$ value & 0.0024 & 0.50 & 1.00 \\
\hline
\end{tabular}




\section{Age with Survivin Status}
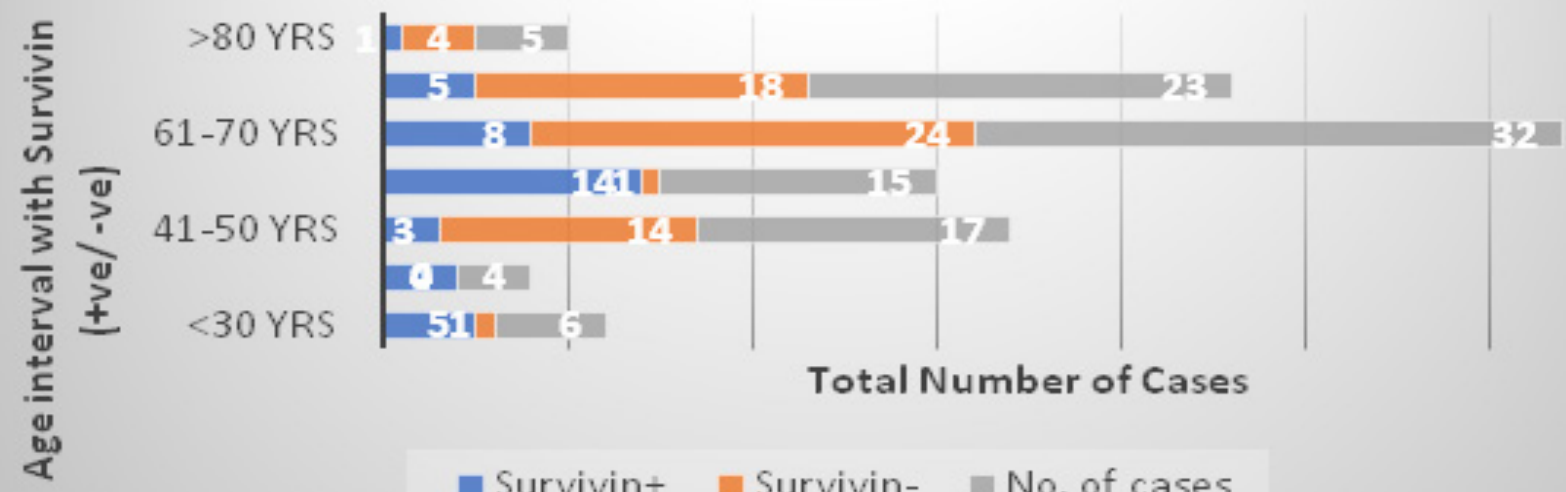

- Survivint Survivin- $\quad$ No. of cases

Fig. 1: Age distribution comparison of Survivin Positive and Survivin Negative tumors.

\section{Gender Distribution}

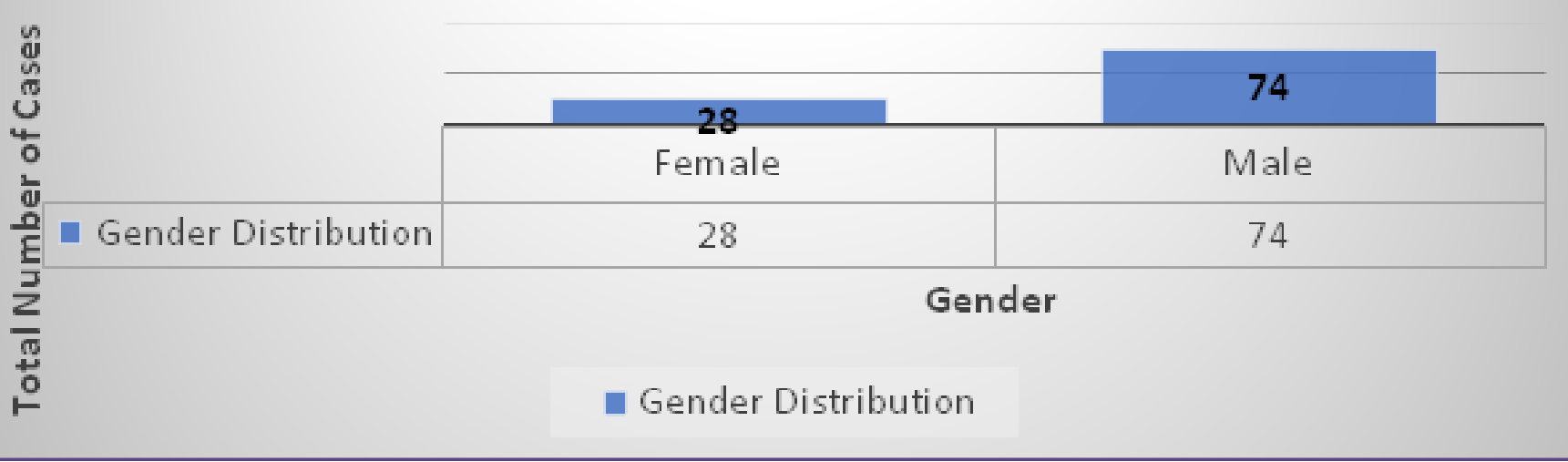

Fig. 1: Age distribution comparison of Survivin Positive and Survivin Negative tumors.

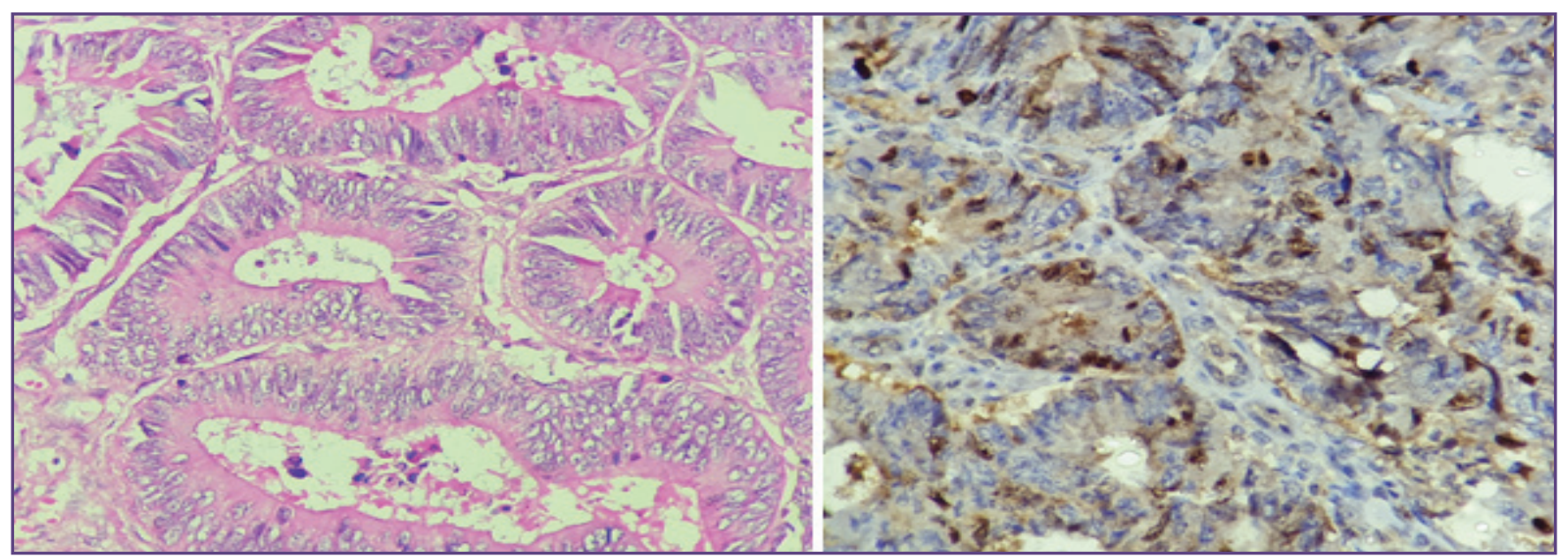

Fig.: 3a: Modeartaely differntiated adenocarcinoma colon (HE 400X), 3b Survivin positivity (IHC 400X). 
DNA microsatellite instability, KRAS mutation, or thymidylate synthase, may be proven to be associated either with prognosis or response to therapy that is independent of TNM stage group or histologic grade. Although these factors are not included in the TNM Summary or Working Stages at this time, several groups are studying the interaction of these clinicopathological factors with TNM and other prognostic factors. ${ }^{[10]}$

Apart from $\mathrm{T}$ and $\mathrm{N}$ category, a range of patient- and tumour-related factors including tumour location and lymph node yield has been associated with survival in stage I-III colon cancer. Lymph node yield beyond the recommended 12 lymph nodes has been associated with improved survival stage I-III colon cancer. Both subsite in the right colon, as well as subsite in the left colon, turned out with adverse prognostic outcome questioning a simple classification into right-sided and left-sided colon cancer. $^{[11]}$

Several studies have suggested immune cells as a promising source of novel diagnostic and prognostic biomarkers by pointing the influence of the immune microenvironment on development of colon cancer. Extent of tumor-infiltrating lymphocytes has been confirmed as an important supplemental marker to the TNM staging system for relapse and mortality prediction. ${ }^{[12]}$ Both the AJCC7 and AJCC8 defined a tumor deposit as a discrete focus of tumor within the lymph node drainage area of the primary carcinoma with no identifiable lymph node. ${ }^{[13]}$

Up to now, only few studies have investigated the molecular profile of pTNM stage I CRC. According to those studies, the percentage of mutated tumors ranges between $25 \%$ and $57 \%$, and mutations mainly involve the KRAS gene in this subset of CRCs. The analysis of KRAS/PIK3CA mutational status may be used to identify patients with stage I CRC at high risk of bad outcome and who may need additional treatments, including biological therapies. ${ }^{[14]}$

In the wake of the 21st anniversary of its discovery, our knowledge of survivin has expanded exponentially, but we are yet to have a survivin-specific anti-cancer agent. ${ }^{[15]}$ However, now that we know survivin is an extraordinary molecule, it is heartening that the development of drugs and peptides that target protein-protein interactions are gathering momentum. Thus, now, with a relatively comprehensive understanding of survivin functions and a molecular inventory of its interactome, it seems that we are in a strong position to make headway in preventing this mischievous little protein from doing what it must. Survivin is a known antiapoptotic protein which enhances the life of a malignant cell thereby making the cancer cell immortal. ${ }^{[16]}$
Survivin, a 142-amino acid, 16.5-kDa protein coded by a single-copy gene BIRC5 (baculovirus inhibitor of apoptosis repeat) located on the human $17 \mathrm{q} 25$ chromosome, is the smallest member of the IAP family. Structurally, Survivin contains a single repeat of the characteristic baculovirusinhibitor of apoptosis domains that are indispensable for the caspase-inhibitory function. ${ }^{[17-21]}$

Regulation of cell division is the main function of Survivin. Normal cells show cell cycle dependent synthesis, expression and degradation of Survivin. Survivin forms a vital component of chromosomal passenger complex (CPC). This CPC ensures proper separation of chromosomes and cytokinesis during cell division. CPC is a hetero-tetrameric complex which localizes to different sites at different times during mitosis. This aids to regulate key events in cell division such as chromosome-microtubule attachment, proper spindle assembly and occurrence of cytokinesis. ${ }^{[22]}$ Survivin when overexpressed, inhibits both intrinsic and extrinsic pathways of apoptosis. Diminution of Survivin in human cells induces defects in apoptotic pathway and cell division. [22] Direct and indirect binding of Survivin to initiator or effector caspases is hypothesized to contribute to inhibition of apoptosis by Survivin. ${ }^{[23]}$ One of the pathways responsible for Survivin facilitated tumor progression is due to angiogenesis in cancer cells. Survivin increases the expression of VEGF and promotes endothelial cell (ECs) proliferation. ${ }^{[24]}$

Survivin induced VEGF expression also helps the tumor cells to be chemo-resistant by stimulating organization of tubulin into distinct fibres. Upregulation of Survivin on tumor vascular ECs as compared to normal tissues, confers drug resistance on tumor vascular ECs. ${ }^{[25]}$ Therefore, targeting Survivin in tumor will promote tumor cell death along with sensitization of cells of tumor vascular network to chemotherapeutic drugs. Role of Survivin has been implicated in regulation of adult stem cell such as in haematopoietic stem cells, neuronal stem cells or intestinal stem cells along with embryonic stem cell and totipotent stem cell. ${ }^{[26][27]}$ As normal stem cells and cancer stem cells have similarities, it can be assumed that like normal stem cells, Survivin expression on cancer stem cells may be implicated in regulating cancer stem cell behaviour. Interestingly Survivin regulates functions of various immune cells, hence therapeutic approaches aimed at targeting Survivin is the need of the hour. Survivin plays a significant role in tumor cell division, apoptosis, chemo resistance and cancer stem cell survival. ${ }^{[28]}$ Various strategies have been envisioned to block Survivin expression in tumour cells (i) Immunotherapeutic approaches to induce immune response against Survivin (ii) small molecule inhibitors/antagonists to block function of Survivin (iii) 
nucleic acid-based approaches which interfere with Survivin gene expression or (iv) gene ablation of Survivin to regulate cell cycle and apoptosis.

We hypothesized that the increased Survivin IHC positivity would indicate worse prognosis in cancer colon patients. We studied the positivity of survivin against the known prognostic variables like- stage, grade, lymphovascular invasion and perineural invasion. In our study, we had 102 cases, of which 76 were Survivin negative whereas 26 cases were Survivin positive. The prevalence of Survivin positivity in cases of carcinoma colon was $25.49 \%$. On statistical analysis we found significant association of survivin positivity with (i) lower age at diagnosis, (ii) differentiation of tumor, (iv) N0 disease(Lymph node status), (v) lymphovascular invasion, (vi) perineural invasion, (vii) group stage. These results were partly concordant with the information published in the literature. There were other variables which were found to be significantly related to the occurrence of Survivin positive tumors like poor differentiation, lymphovascular invasion, perineural invasion, group stage in this study which were not mentioned in other studies under reference or were not significant. Our study inferred that survivin positivity is significantly associated with higher grade, higher stage, LVI positivity and PNI positivity. However, on performing multivariate analysis we found significant association of survivin positivity with age only. This could be attributed to the ethnic and genetic differences in the population studied as, in the literature review, we could only come across the studies done on the western world population. Thus, in this case, the comparison of data may not be entirely appropriate.

\section{Conclusion}

In spite of this limitation, our study recommends performing Survivin IHC in small initial biopsies of suspected colon cancer cases. The positivity of Survivin in the initial biopsy can predict a worse outcome. In future as envisaged the advent of targeted therapy against Survivin shall go a long way in treating cases of colon cancer.

\section{Acknowledgments}

Nil

\section{Funding}

Nil

\section{Competing Intrest}

Nil

\section{References}

1. Ali I, Wani WA, Saleem K. Cancer Scenario in India with Future Perspectives. 2011;8:56-70.
2. Divitiis C De, Nasti G, Montano M, et al. Prognostic and predictive response factors in colorectal cancer patients : Between hope and reality. 2014;20(41):15049-15059.

3. Press D. Prognostic stratification of colorectal cancer patients : current perspectives. 2014:291-300.

4. Khan Z, Khan AA, Yadav H, Prasad GBKS, Bisen PS. Survivin, a molecular target for therapeutic interventions in squamous cell carcinoma. 2017:1-32

5. Jaiswal P, Goel A, Mittal R. Survivin: A molecular biomarker in cancer. Indian J Med Res. 2015;141(4):389-397.

6. Taubert H, Heidenreich C, Holzhausen H-J, et al. Expression of survivin detected by immunohistochemistry in the cytoplasm and in the nucleus is associated with prognosis of leiomyosarcoma and synovial sarcoma patients. BMC Cancer. 2010;10:65.

7. Amin MB, Greene FL, Edge SB. The Eighth Edition AJCC Cancer Staging Manual: Continuing to build a bridge from a population-based to a more "personalized" approach to cancer staging. CA Cancer J Clin. 2017;67(2):93-99.

8. Sarela GK. Expression of the antiapoptosis gene, Survivin, predicts death from recurrent colorectal carcinoma. Gut. 2000;46(5):645-650.

9. Kawasaki, Hiroshi, et al. "Inhibition of apoptosis by survivin predicts shorter survival rates in colorectal cancer." Cancer research 58.22 (1998): 5071-5074

10. Hamilton SR, Aaltonen LA. World Health Organization Classification of Tumours Pathology and Genetics of Edited by. 2000 .

11. Lykke J, Rosenberg J, Jess P, Roikjaer O. Lymph node yield and tumour subsite are associated with survival in stage I-III colon cancer: Results from a national cohort study. World J Surg Oncol. 2019;17(1):1-8.

12. Zhou R, Zhang J, Zeng D, et al. Immune cell infiltration as a biomarker for the diagnosis and prognosis of stage I-III colon cancer. Cancer Immunol Immunother. 2019;68(3):433-442.

13. Landau MA, Zhu B, Akwuole FN, Pai RK. Histopathological Predictors of Recurrence in Stage III Colon Cancer: Reappraisal of Tumor Deposits and Tumor Budding Using AJCC8 Criteria. Int J Surg Pathol. 2019;27(2):147-158.

14. Bonetti LR, Barresi V, Maiorana A, Manfredini S, Caprera C, Bettelli S. Clinical impact and prognostic role of KRAS/ BRAF/PIK3CA mutations in stage I colorectal cancer. Dis Markers. 2018;2018, 2959801.

15. Wheatley SP, Altieri DC. Survivin at a glance. J Cell Sci. 2019;132(7):223826.

16. Lazo JS, Sharlow ER. Drugging Undruggable Molecular Cancer Targets. Annu Rev Pharmacol Toxicol. 2016;56:23-40.

17. Deveraux QL, Stennicke HR, Salvesen GS, Reed JC. Endogenous inhibitors of caspases. J Clin Immunol. 1999;19(6):388-398.

18. Uren AG, Wong L, Pakusch M. Survivin and the inner centromere protein INCENP show similar cell-cycle localization and gene knockout phenotype. :1319-1328. 
19. Li F, Yang J, Ramnath N, Javle MM, Tan D. Nuclear or cytoplasmic expression of survivin: what is the significance? Int J cancer. 2005;114(4):509-512.

20. Dohi T, Beltrami E, Wall NR, Plescia J, Altieri DC. Mitochondrial survivin inhibits apoptosis and promotes tumorigenesis. J Clin Invest. 2004;114(8):1117-1127.

21. Khan S, Bennit HF, Wall NR. The emerging role of exosomes in survivin secretion. Histol Histopathol. 2015;30(1):43-50.

22. Li F, Ackermann EJ, Bennett CF. Pleiotropic cell-division defects and apoptosis induced by interference with survivin function. Nat Cell Biol. 1999;1(8):461-466.

23. Antonio CH, Tsai F, Lee JY. Survivin - biology and potential as a therapeutic target in oncology. 2013:1453-1462.

24. Fernandez JG, Rodriguez DA, Valenzuela M. Survivin expression promotes VEGF-induced tumor angiogenesis via PI3K/Akt enhanced beta-catenin/Tcf-Lef dependent transcription. Mol Cancer. 2014;13:209.
25. Virrey JJ, Guan S, Li W, Schonthal AH, Chen TC, Hofman FM. Increased survivin expression confers chemoresistance to tumor-associated endothelial cells. Am J Pathol. 2008;173(2):575-585.

26. Hoggatt J, Singh P, Sampath J, Pelus LM. Prostaglandin E 2 enhances hematopoietic stem cell homing, survival and proliferation. 2017;113(22):5444-6

27. Mull AN, Klar A, Navara CS. Differential localization and high expression of SURVIVIN splice variants in human embryonic stem cells but not in differentiated cells implicate a role for SURVIVIN in pluripotency. Stem Cell Res. 2014;12(2):539-549.

28. Gurbuxani S, Xu Y, Keerthivasan G, Wickrema A, Crispino JD. Differential requirements for survivin in hematopoietic cell development. Proc Natl Acad Sci U S A. 2005;102(32):11480-5.

*Corresponding author:

Gp Capt Prateek Kinra, Dept. Of Pathology, AFMC, Wanowrie, Pune- 411040

Phone: +919945277110

Email: pkinra_in@yahoo.com

Date of Submission : 07/07/2020

Date of Acceptance : 02/10/2020

Financial or other Competing Interests: None.

Date of Publication : 30/11/2020 